being potentially highly vulnerable to exploitation; b) increasing civil society engagement and improving uptake of the ethics dimension in research and innovation; and c) improving global awareness of the highest ethical standards along with clear, defined incentives for research competitiveness.

\section{OC-8724 NEW VACCINES FOR A SAFER WORLD}

Nathalie Imbault*, Rachel Grant. CEPI, Oslo, Norway

10.1136/bmjgh-2019-EDC.46

CEPI is an innovative partnership between public, private, philanthropic and civil organisations founded in Davos in 2017 to develop vaccines to stop future epidemics. To date, CEPI has received multi-year funding from Norway, Germany, Japan, the Bill \& Melinda Gates Foundation, and the Wellcome Trust. CEPI has also received single-year investments from the governments of Australia, Belgium, and Canada. It has reached $\$ 630$ million of its $\$ 1$ billion funding target. The European Commission has announced a contribution in kind of $€ 250$ million that will support relevant projects through EC mechanisms. Since its launch in January 2017, CEPI has announced two Calls for Proposals. The first was for candidate vaccines against MERS-CoV, Nipah and Lassa viruses. The second was for the development of platforms that can be used for rapid vaccine development against unknown pathogens. From the first call, CEPI has, so far, announced six partnership agreements and this means 4 lassa vaccine candidates, 3 MERS-CoV vaccine candidates and 1 Nipah vaccine candidate have been selected for development. Learn more at CEPI.net. Follow us at@CEPIvaccines.

\section{ABSTRACTS OF POSTER PRESENTATIONS}

\section{PO 7139 CONDITIONAL ECONOMIC INCENTIVES AND MOTIVATIONAL INTERVIEWING TO IMPROVE ADOLESCENTS' RETENTION AND ADHERENCE TO ANTIRETROVIRAL THERAPY IN NIGERIA: ARA TRIAL}

${ }^{1}$ Obinna Ekwunife, 'Maureen Anetoh, ${ }^{2}$ Stephen Kalu, 'George Eleje. 'Nnamdi Azikiwe University, Awka, Nigeria; ${ }^{2}$ Nnamdi Azikiwe University Teaching Hospital, Awka, Nigeria

10.1136/bmjgh-2019-EDC.47

Background Adolescent HIV patients have worse treatment outcomes compared to other age groups given their vulnerability to risk-taking behaviour. Limited evidence exists on the effectiveness of service delivery interventions to support adolescents' retention in care and adherence to antiretroviral therapy (ART). The ARA trial tackles this challenge by evaluating the impact of conditional economic incentives coupled with motivational interviewing on adolescents' retention in care and adherence to ART in Anambra State, Nigeria.

Methods The study will be a cluster-randomised, controlled trial conducted in $12 \mathrm{HIV}$ treatment hospitals in Anambra State, Nigeria. Six (6) hospitals each will be randomised to either intervention or control arm. A structured adherence support scheme, termed the Incentive Scheme, will be applied to the intervention hospitals while the control hospitals will receive routine HIV care. Additionally, patients in the intervention arm will receive motivational interviewing at baseline and following initiation of ART, they will receive a gift voucher of Nigerian Naira (NGN) 2000 when viral load (VL) is $<20$ copies $/ \mathrm{mL}$ at 12 weeks, gift voucher of NGN 1000 if the VL remains suppressed for the next 3 months, and the next 6 months, and finally gift voucher of NGN 2000 if the $\mathrm{VL}$ remains $<20$ copies/mL at 1 year. All gift vouchers will be conditional not only on VL results but also on attending for motivational interviewing. The primary outcome for the trial will be the difference between groups in the proportion to HIV viral load suppression $(\leq 20$ copies/mL) by 12 months and then 24 months after withdrawal of the Incentive Scheme.

Results The protocol for ARA trial and planned activities is finalised. Application for approval for the trial is on-going.

Conclusion The proposed trial will provide evidence on the feasibility of applying the Incentive Scheme to improve retention and adherence to ART of adolescents living with HIV.

\section{PO 7151 COMPARING TWO BED NET DELIVERY MODELS IN RURAL DISTRICTS OF MOZAMBIQUE}

Jorge Arroz. Mozambique Medical Council, Maputo, Mozambique

\subsection{6/bmjgh-2019-EDC.48}

Background The use of long-lasting insecticidal nets (LLINs) is associated with a reduction in malaria transmission. In 2015, a new delivery strategy (intervention) for universal coverage campaign was tested and compared with standard strategy (control). The objective is to compare two bed net delivery models in rural districts of Mozambique.

Methods Two districts served as intervention, and two as control. The following study design was used: 1) before and after; and 2) cost-effectiveness analysis. Three core implementation strategies were tested: 1) use of coupons during household registration, 2) use of stickers to identify registered houses, and 3) a new LLINs allocation criterion. The main endpoints measured were: i) percentage of distributed LLINs; ii) LLINs ownership and use coverage; iii) percentage of households that achieved universal coverage; iv) incremental cost-effectiveness ratio (ICER); v) incremental net benefit (INB).

Results Approximately 88\% $(302,648)$ of LLINs were distributed in intervention districts compared to $77 \%(219,613)$ in control districts [OR: 2.14 (95\% CI: 2.11-2.16)]. Six months after the 2015 campaign, $98.8 \%$ of the 760 households surveyed in the intervention districts had at least one LLIN; $89.6 \%$ of the 787 households surveyed in the control districts had at least one LLIN [OR: 9.7, (95\% CI: 5.25-22.76)]. Near $95 \%$ and $87 \%$ of respondents who had at least one LLIN, reported having slept under the LLIN the previous night in the intervention and control districts, respectively [OR: 3.2; (95\% CI 2.12-4.69)]; 71\% of the households surveyed achieved universal coverage in the intervention districts against $59.6 \%$ in the control districts [OR: 1.6; $(95 \% \mathrm{CI}$ : 1.33-2.03)]. ICER per distributed LLIN was US\$ 0.68. INB was positive.

Conclusion Intervention districts had greater LLINs availability, greater LLINs ownership and use coverage, and a better progression toward reaching universal coverage targets. The new strategy was more cost-effective than the previous strategy. 